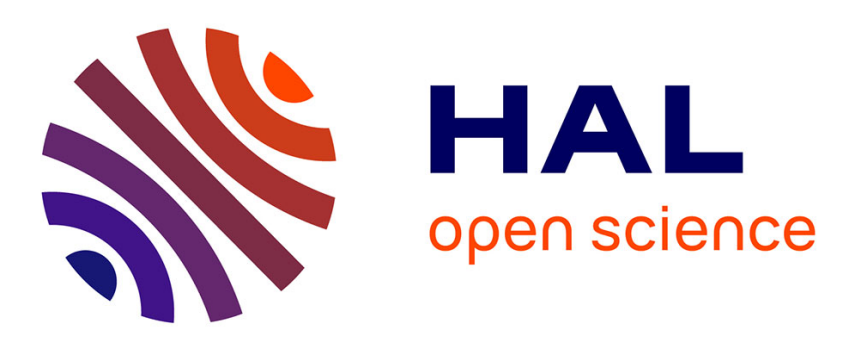

\title{
A Smart Spinal Orthopedic Bed for General Purpose Rehabilitation
}

\author{
Hanin Elsayed, Nadine Ghannam, Amira Zaylaa
}

\section{To cite this version:}

Hanin Elsayed, Nadine Ghannam, Amira Zaylaa. A Smart Spinal Orthopedic Bed for General Purpose Rehabilitation. The 5th International Conference on Advances in Biomedical Engineering, IEEEEMBS, Oct 2019, Tripoli, Lebanon. inserm-02320946

\section{HAL Id: inserm-02320946 https://www.hal.inserm.fr/inserm-02320946}

Submitted on 19 Oct 2019

HAL is a multi-disciplinary open access archive for the deposit and dissemination of scientific research documents, whether they are published or not. The documents may come from teaching and research institutions in France or abroad, or from public or private research centers.
L'archive ouverte pluridisciplinaire HAL, est destinée au dépôt et à la diffusion de documents scientifiques de niveau recherche, publiés ou non, émanant des établissements d'enseignement et de recherche français ou étrangers, des laboratoires publics ou privés. 


\section{A Smart Spinal Orthopedic Bed for General Purpose Rehabilitation}

\author{
Hanin ElSayed, Nadine Ghannam, Amira J. Zaylaa \\ Department of Biomedical Engineering, \\ Lebanese International University, Bekaa, Lebanon, \\ Email: 21730439/21630914@students.liu.edu.lb, \\ amira.zaylaa@liu.edu.lb
}

\author{
Amira J. Zaylaa \\ Neoroscience Research Center, \\ Faculty of Medical Sciences, Lebanese University, \\ Faculty of Public Health-V, Lebanese University, \\ Lebanon, \\ Email: amira.zaylaa@ul.edu.lb
}

\begin{abstract}
Critical spinal cord injuries and paralyzation are amongst the leading medical concerns. Lack of movement associated with post-operative phases and paralization complicates the lives of patients from one side, and subject the nurses and families to an excessive load from the other side. Despite the presence of Stryker and one function orthopedic beds for rehabilitation purposes, they were either expensive or non-automated. Thereby, it was not prevailing to see these beds in hospitals and/or houses as assistive tools. The aim of our innovative and exploratory study is to design a smart orthopedic bed for patients, starting with children, in order to facilitate their movement while maintaining their posture. Rotation was considered laterally from one side to another while maintaining their body fixed. The smart bed was automatically controlled by sound recognition, and manually controlled through a touchscreen for safety. Simulation results showed the best fit and architecture of the building up parts of the smart bed. Experimental results revealed the compact design, the touchscreen with its facilities, and various immobility functions to provide feasibility while performing daily activities. The feasibility of triggering the smart system reduced the time deducted from nurses and family members. Statistical Evaluation showed that the smart design was $\mathbf{9 5 \%}$ efficient in performing the movement as opposed to the Stryker $(55 \%)$ and one function orthopedic beds $(45 \%)$. The net cost of the system was low $(\$ 775)$ as opposed to alternatives $(\mathbf{\$ 1 0 0 0 - 1 3 0 0 )})$. The time of rotation of the smart bed was in seconds. As a future prospect more complicated movements could be considered to provide specific types of rehabilitation for the spinal cord.
\end{abstract}

Index Terms-Smart Orthopedic Bed; Spinal Cord Injuries; Rehabilitation; Biomedical Instrument; Statistical Evaluation.

\section{INTRODUCTION}

Critical spinal cord damage is the harm to any part of the spinal cord or nerves at the end of the spinal canal, it frequently causes permanent modifications in strength, sensation and different body features. The ability to control the limbs after a spinal cord injury depends on two factors: the place of the injury along the spinal cord and the severity of injury in the spinal cord. Spinal cord injuries may result from the damage of the vertebrae or disks of the spinal column, accidents, falling, violence, diseases etc. [1], [2]. Spinal cord injuries may result in various symptoms such as, loss of movement and difficulty in stability and walking, lower back ache, loss of bladder control, exaggerated reflex activities and others [1].
As a rehabilitation and support, Kinetic therapy was used to mobilize severely disabled humans by means of placing them on a distinctive rotating bed (orthopedic beds). This remedy has the unique capability of anatomically immobilizing acutely ill patients, while developing a state of relative physiological mobility. Kinetic therapy provided preliminary proof for its efficiency as a rehabilitation method [2]. Consequently, the revolution in beds started for this therapy, when first the "one function" orthopedic bed was designed, which was mainly used for hips surgeries especially for babies and children. However, the one function orthopedic bed was not capable of tackling adults and elder patients. Later, many bed designs were published under the marketing name "orthopedic", however, these mattresses where either too soft or too firm, thus no support for pressure points where supplied, also they affected directly the comfort of the body curvature and the spine when they are bent for a period of time. Recently, Stryker frame bed was the final method invented to assist patients, this bed allows the movement of body without disrupting the traction but it requires a lot of time to be rotated and it employs two or more nurses to apply rotation.

To this purpose, the aim of our innovative and exploratory study is to design an automated Smart spinal orthopedic bed that facilitates the movement of the patient, starting with the children, in order to reduce their pain, supply them with the optimal balanced mattresses, and reduce the time of nurses and family members [3], [4].

The paper is organized as follows. Section I provides a summary of the therapeutic and rehabilitation methods and the aim of our innovative and research work. Section II presents the materials and the Smart Orthopedic Bed design. Section III showcases the simulated, experimental and statistical evaluation results of the smart design. Section IV provides the discussion of the Smart Orthopedic Bed as opposed to alternatives and the conclusion, and section $\mathrm{V}$ provides the future work.

\section{SMARt ORthopedic Bed Materials}

The major parts of the smart orthopedic bed design are summarized in the block diagram shown in Fig. 1. The servomotors, relays, Arduino Mega, voice recognition module, load cell, touchscreen, leds, Plexiglas, and an Adapter (12 $\mathrm{V}, 3 \mathrm{~A}$ ) 


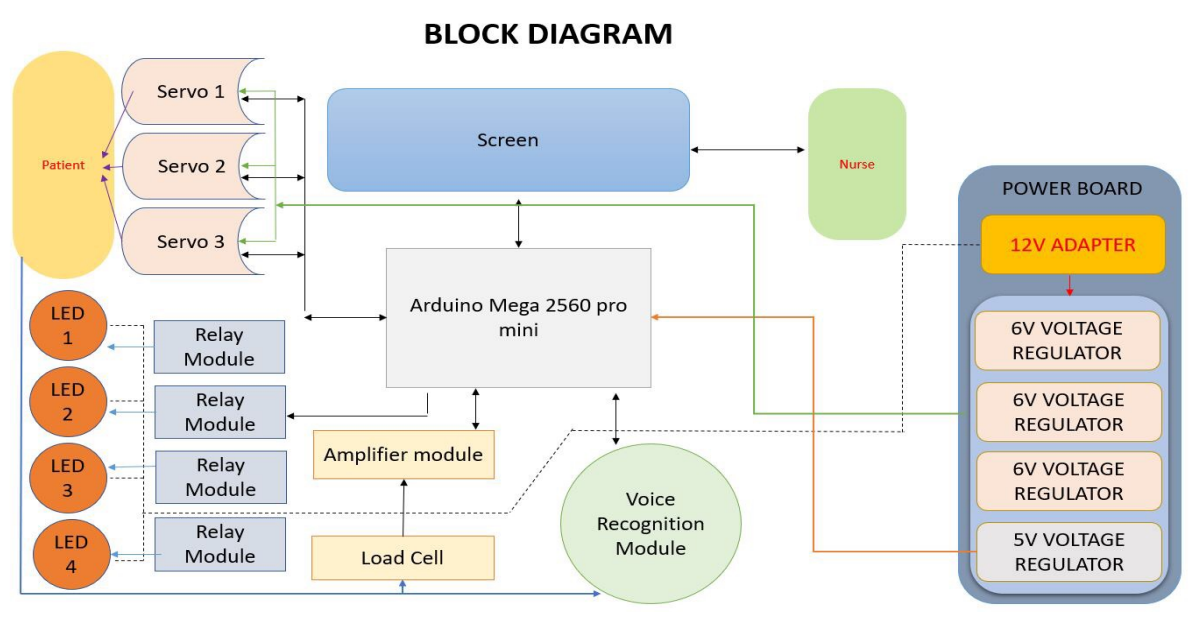

Fig. 1: The Block Diagram of the Smart Orthopedic Bed.

were used. The servomotors were used as they form closedloop servomechanism that uses position feedback to control both the motion and position. The relay was used as an automatic switch, it transforms $5 \mathrm{~V}$ to $12 \mathrm{~V}$ to light up the leds. The Arduino was used to control all the other modules. The Arduino takes the order from the screen and sends the signal to the motor to rotate. It also takes data from the load cell and sends it to the screen. The voice recognition module was used, as it takes the order from the sound through the microphone that amplifies and processes the sound. Some words were saved by the code so that the module recognizes them. The load cell was chosen to work on the variation of the resistance when a body is placed on the bed. The weight can be measured according to a built in equation in the library of load cell. The touchscreen, was used to control the system and reads the data, such as the torque and the weight. The leds were used on all the bed sides as they lighten up in the direction ordered when the relay supply them with $12 \mathrm{~V}$. The Plexiglas was used to design the bed structure. An Adapter $(12 \mathrm{~V}, 3 \mathrm{~A})$ was used to supply leds and servomotors with voltage. Finally, a 7806 voltage regulator was used to regulate the voltage to $6 \mathrm{~V}$ after a $12 \mathrm{~V}$ was taken from the adapter, and to supply the servomotor with the regulated voltage [5].

\section{Smart Orthopedic Bed Methodology}

The method followed along with the requirements including the (i) touchscreen methodology and (ii) interactions were provided. In the (i) touchscreen methodology, the system works based on the screen connected to it. When the system is turned on, the Log-in page appears. After entering the correct Username and password, the nurse can enter the patient's name, ID, and room number. The menu page appears then the bed settings which can control the bed directions. The weight, torque and angle of each motor was designed to appear on the screen. The nurse can control each motor and the angle of motion. Voice recognition settings can be enabled or disabled through the screen. In the (ii) interactions:

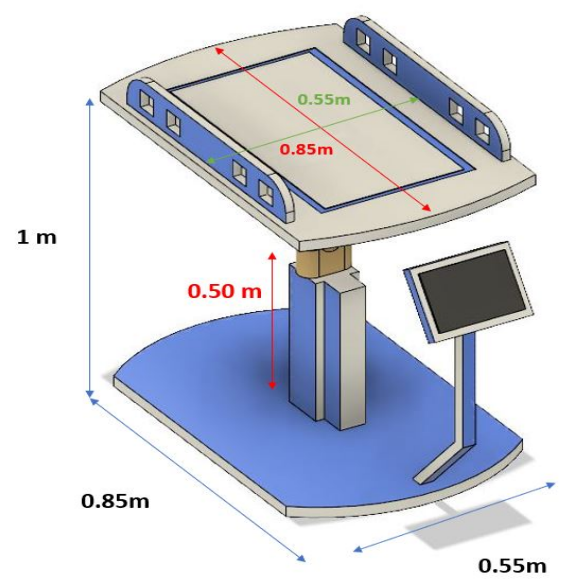

Fig. 2: The Simulated Form and Dimensions of the Smart Spinal Orthopedic Bed.

- Screen-Motor interaction: the screen is connected to the Arduino by the receiver RX and transmitter TX wires, respectively. When the order is replaced, it is sent through TX wire of the screen, and received by the RX wire of the Arduino. After taking the order from the screen, it gives a signal to one of the digital PINS of the motors; the motor that receives the signal applies the order.

- Load cell-Arduino-Screen interaction: the load cell sends the mass of the patient by its TX wire which is received by RX wire of the Arduino.

- Voice-recognition module-Arduino-Motor: the module is connected to the Arduino, RX of module is connected to TX1 of Arduino and TX of the module is connected to the RX1 of the Arduino, i.e. when the patient talks, the module sends a signal from its RX to the TX of Arduino which then sends the order to either move the motors or request the nurse.

In the testing process, we first started by testing each component alone to make sure it was functioning well. Then we connected all the components to form the required system 


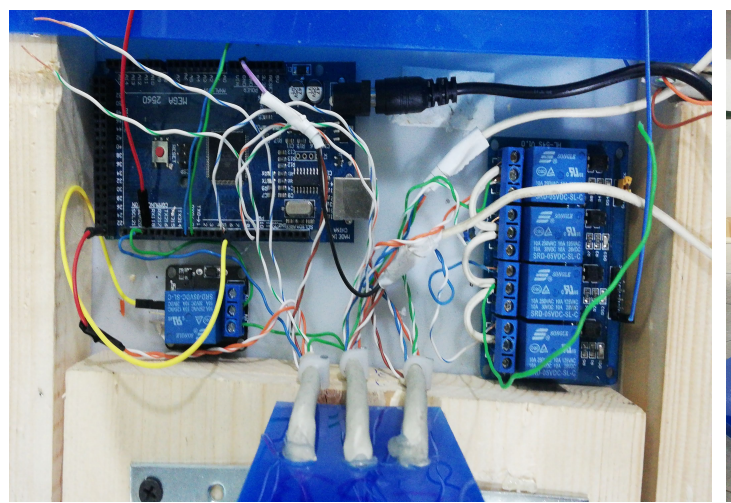

(a)

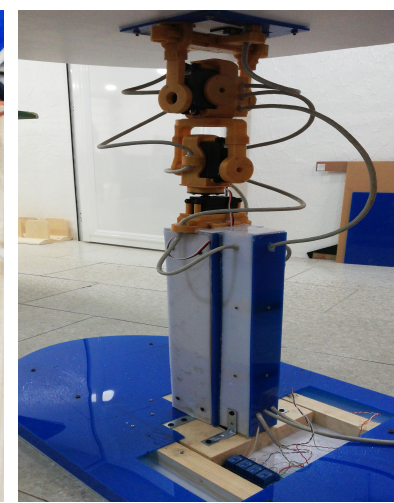

(b)

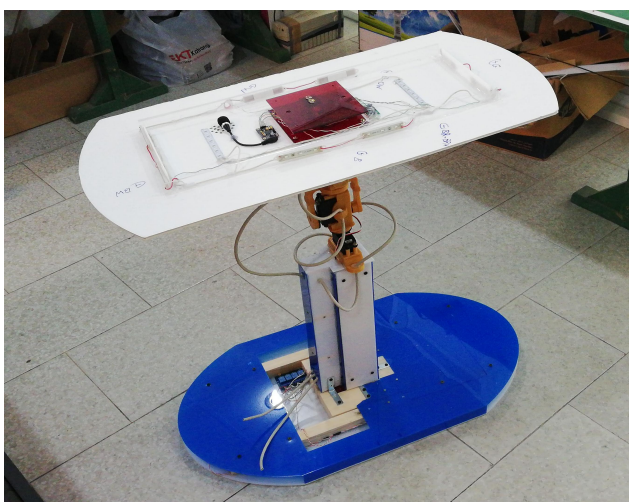

(c)

Fig. 3: The Preliminary Design of the Smart Orthopedic Bed. (a) The Arduino Connections. (b) The Attached Servomotors. (c) The Architecture of the Smart Orthopedic Bed.

while testing was in progress. We coded each component, and finally we combined all the codes side by side to get the final system working. The Arduino was tested for controlling and giving orders from the components connected to it. The link between the screen and Arduino is both ways, i.e. the screen gives and takes the order to and from the Arduino. For instance, the nurse gives order to rotate the bed through the Arduino and servomotors. On the other hand, the Arduino takes the mass of the patient from the load cell and sends it to the screen. The screen is the only component that takes and gives orders, while the other components only take orders from the Arduino.

\section{SMART ORThopedic Bed Results}

Herein, the smart orthopedic bed results are divided into simulation, experimental and evaluation results.

\section{A. Simulation Results}

The AutoCAD simulation was used to draw the planned geometrical aspects of the smart orthopedic bed. The simulation shows the architecture contribution illustrated in Fig. 2. The system was made up of three main parts: the base, body, and top. The base was made up of Plexiglas containing the circuit, which connects some of the electronic parts of the system. The body comprised three 3-D printed servomotors. And the top was made up of Plexiglas, containing the load cell leds and voice recognition module. The top also comprised supporting parts that hold the belts supporting the patient.

\section{B. Experimental Results}

In order to get the bed to rotate and achieve all the tasks required, a system of circuits connected to the Arduino was made as shown in Fig. 3 (a). The orthopedic bed rotates 360 degrees by using three servomotors to ensure the rotation and movement. The first servomotor rotates the bed on X-Y plane (left and right), while the second rotates the bed on Y-Z plane and the third motor on $\mathrm{X}-\mathrm{Z}$ plane. A real picture was taken as shown in Fig. 3 (b) of the 3 motors fixed on 3-D printed parts, and the body in as shown in Fig. 3 (c).

\section{Experimental Evaluation}

In order to make sure that the smart orthopedic bed was the best seller in the market, a comparison bar graph was made and reported in Fig. 4. We compared the smart orthopedic bed to the Stryker frame bed and the one function orthopedic bed according to the efficiency as shown in Fig. 4 (a), the time the bed takes to rotate 5 degrees as shown in Fig. 4 (b), the number of features that each bed has as shown in Fig. 4 (c), and the cost of the smart bed as opposed to the alternatives as shown in Fig. 4 (d). Noteworthy that the efficiency was calculated as the power of the motor divided by total power of the motor. The servomotors used in this prototype were high torque servomotors placed above each other to give the bed a uniform movement in all the directions; also this technique decreases the distance between the motor and the bed so that the torque decreases. The efficiency of the bed was evaluated by placing a mass on different positions on the bed and testing the torque of each motor, this was repeated 15 times. The smart orthopedic bed achieved the best results with a minimal cost unlike other designs. The net cost is low and affordable as illustrated in Fig. 4 (b).

According to statistical evaluation, the efficiency of moving the angle of the bed is equal to the efficiency of the servomotor, i.e. 90 degrees $=95 \%$ according to Fig. 4 (a). In addition, the bed's electronic and mechanical safety was considered. Our system was powered by $5 \mathrm{~V}, 6 \mathrm{~V}$ and $12 \mathrm{~V}$ DC and was categorized as a class 2 safety. The servomotors had high torque that limits the moving angle of the servo. Our system was mostly made up of plastic and Plexiglas to avoid the conduction of electricity, thus it is safer for the patient. To increase the safety and secure the information of the patient, a screen was implemented with a login page that required a username and a password to ensure a professional control of the bed.

\section{Discussion AND CONCLUSION}

The smart orthopedic bed was successfully developed and was functioning in a way that can fulfill the patient's needs. 


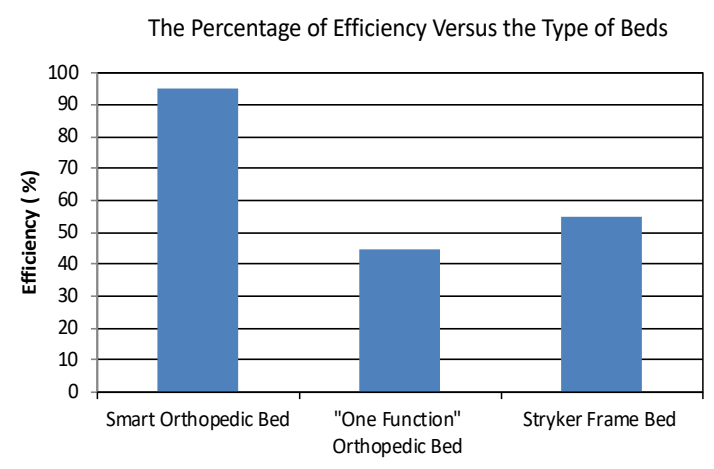

(a)

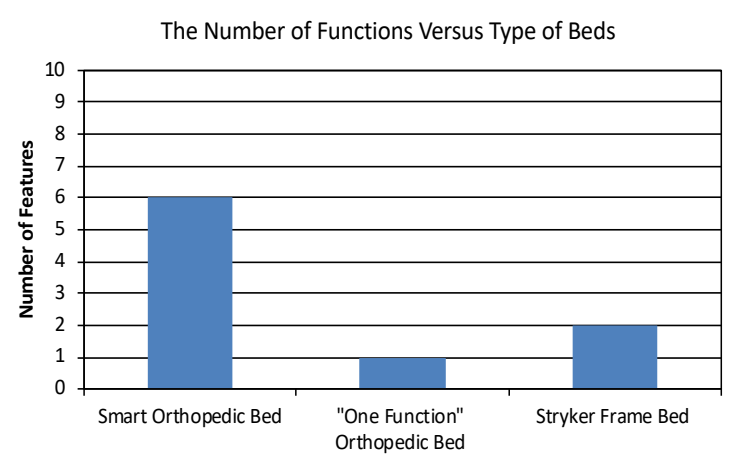

(c)

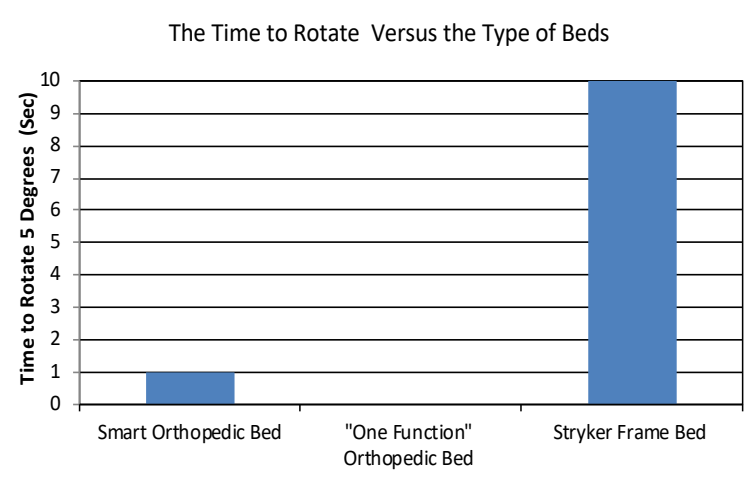

(b)

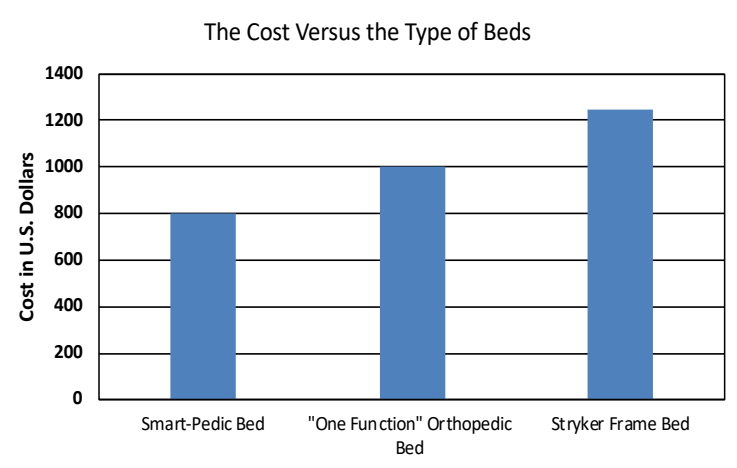

(d)

Fig. 4: The Variation of Features Relative to the Three Beds Discussed. (a) The Percentage of Performance Versus the Type of Beds. (b) The Cost Versus the Type of Beds.

Our design was suitable for extension to serve adults and infants as opposed to the orthopedic bed that was invented in 1741. Moreover, our design surpassed the design invented in 1950 tackling mattresses, which was neither a firm mattress providing comfort for pressure points nor a soft surface provided support. We supported our design with a mattress that has the optimal balance of softness and support.

Last but not least, Stryker frame bed invented by H. Stryker was based on joining two disks around each other to help rotate the bed manually which wastes time, requires energy and requires the nurse's strength [6]. However, our smart spinal orthopedic bed allowed the movement of the body without any disruption of traction; and since our design was controlled by a screen connected to the Arduino and a voice recognition module, the time of rotation was reduced without the need of others. Besides, our design was considered as a vast option for injured patients, because of its flexible and comfortable structure and affordable cost.

Our multi-function orthopedic bed is a design which meant to provide several features and movement which was not taken in consideration in other beds. The smart orthopedic bed was designed to function with a touchscreen connected to the bed to make it feasible for the nurse to deal with. As well as the bed comprised a voice recognition system to allow the patient to control it. This bed offers 360 degrees' rotation; it flips sideways to maintain a normal blood flow, and it stands in a steady position to decrease stress and strain on the patient's back pressure points. The bed was of low cost and surpassed alternatives in the number of features it provides, the efficiency and the time of rotation.

\section{FUTURE WORK}

As a future prospect we could include in our smart orthopedic bed the idea of flipping the bed upside down, and making the mattress separated from the patient, in case the patient needs some physical therapy or needs to change his cast. We could also evolve the mattress to aid in wounds caused while lying in the bed for a long time by adding moisturizer and temperature sensors. Moreover, the extension of the size of the bed will be considered to make it usable not only by children but also by adults.

\section{REFERENCES}

[1] D. K. Zhao, "Spinal cord injury, mayo foundation for medical education and research," [online], May 2019, https://www.mayo.edu/research/centers-programs/spinal-cord-injuryresearch-program.

[2] T. N. Byrne, C. E. Benzel, and G. S. Waxman, Diseases of the Spine and Spinal Cord. Oxford University Press, 2000, vol. 58.

[3] J. Auer, "Mechanics of orthopedic mattresses and how its largely used as marketing term," Matress Clarity, 2015.

[4] H. H. Stryker, "Stryker frame," [Online], 2009, farlex and Partners, https://medical-dictionary.thefreedictionary.com/Stryker+frame.

[5] B. A. Green, K. L. Green, and K. J. Klose, "Kinetic therapy for spinal cord injury," Spine, vol. 8, no. 7, pp. 722-728, 1983.

[6] T. Thinnes, "Homer stryker and his revolutionary bed," Museography, vol. 2 , no. 1 , pp. 15-24, 2002. 